\title{
High-Energy Radiation Dosimetry in the Space
}

\section{Sahare PD* $^{*}$}

Department of Physics \& Astrophysics, University of Delhi, India

In recent years there has been much more increase in the air traffic and space expeditions. However, the terrestrial space is not as safe as the Earth's surface or the atmosphere surrounding the planet Earth due to presence of high-energy particles and radiations. There is some background radiation on the surface or in its surrounding atmosphere too but it is less harmful and generally much less than the limit (prescribed by International Commission for Radiation Protection (ICRP) and other regulatory authorities such as NCRP) as the Earth's atmosphere acts as a natural radiation shield. Not only aircrew and space-crew but the frequent flyer passengers need to be protected from such hazardous radiations and therefore continuous radiation monitoring is necessary so that the total dose per annum does not exceed the prescribed limit $[1,2]$. There had been many expeditions in the space where the radiation dosimetry was done extensively, e.g. MATROSHKA-R [3,4], FOTON-M2 Satellite [5], MIR space station and the International Space Station (ISS) and many more [6,7]. There are also good reviews on the work could be found in the literature $[8,9]$. According to the National Council on Radiation Protection and Measurements (NCRP) recommendation No. 132 [10], 10 years career limits for stochastic effects (based on 3\% excess lifetime risk of cancer mortality) range from 0.4 to $3.0 \mathrm{~Sv}$, depending on the gender and age.

The radiation environment beyond the Earth's atmosphere consists mainly of galactic cosmic rays (GCR) and trapped particles [5]. When these primary GCRs (may be originating from outside the solar system) reach the upper atmosphere, their interaction with oxygen and nitrogen nuclei produces secondary particles such as neutrons, protons and pions, which, in turn, can either interact further with atmospheric other nuclei or decay radioactively to develop a cascading effect for producing electron-photon pairs, hadronic and muonic particles, etc. For the estimation of doses on board spacecraft or during a space walk, a combination of thermoluminescent detectors (TLDs) and plastic nuclear track detectors (PNTD) are very suitable due to their several advantages, such as light weights and small sizes, no need of power supply and easy data handling. They could also be easily calibrated using phantoms simulating human bodies and could be placed at various locations inside the spacecraft to see variation of doses at different locations [3-7]. Recently developed OSL dosimeters (OSLDs) could be more useful due to the possibility of using them remotely and online.

However, the estimation of high-energy radiation doses in space had never been easy and poses many challenges. Firstly, it is a mixed field consisting of galactic cosmic rays (GCR) and all sorts of secondary trapped particles such as neutrons, protons and pions, electron-photon pairs, hadronic and muonic particles, etc. In such a mixed field it is very difficult to measure the exact dose by a single type of dosimeter due to different interaction cross sections. Secondly, for resolving dose and dose rate in such an environment with very low flux, dosimeters should be capable of determining the ionization density of each incident particle. Neutron dosimetry involves an additional challenge that must be met to accurately estimate the effective dose to aircrews and astronauts [11]. Neutron-sensitive bubble detector may be ideal as a portable personal dosimeter in space but power consumption may be a problem [12]. In addition, there are other constraints such as cost, size, weight, and power consumption, sensitivity and energy response in a mixed field prohibit use of a single type of a dosimeter. Passive dosimetry methods continue to provide the most relevant data, but often require extensive processing after returning to Earth. Active detectors can provide time-resolved data in real time, but size and power consumption prevent these instruments from operating as personnel dosimeters. Due to enormous cost involved in estimating the exposure levels on board aircrafts and spacecrafts theoretical approach involving simulations usually based on the Monte Carlo codes [13] could also be used, however, the reliability of the data depends on the environment and transport models used in such codes.

Along with other high-energy radiations as mentioned earlier, energetic heavy charged particles, may also be found in typical space radiation fields. The space radiation environment consists of a mixture of medium-to high-energy charged particles, from electrons to heavy ions such as Fe, Co, Ni. The 'absorbed dose', however, is mainly due to electrons in the medium to high-energy range and protons having low linear energy transfer (LET), whereas the 'dose equivalent' is determined by both primary and secondary heavy ions with high LET and secondary neutrons [14]. It is, therefore, difficult to use high-Z TLD phosphors, such as, $\mathrm{CaSO}_{4}$ :Dy or $\mathrm{CaF}_{2}$ :Dy in a mixed field as their responses are energy dependent. Low- $\mathrm{Z}$ phosphors such as $\mathrm{LiF}: \mathrm{Mg}, \mathrm{Ti}$ (TLD 100) and LiF:Mg,Cu,P (e.g. TLD 700H) or Li, K, Na-doped BeO (i.e. $\mathrm{BeO}: \mathrm{Li}, \mathrm{BeO}: \mathrm{Na}, \mathrm{BeO}: \mathrm{K}$ ) tissue equivalent phosphors could be used in such an environment. However, they too have some drawbacks as LiF based phosphors loose their sensitivity and reusability on taking TL readout beyond $523 \mathrm{~K}$ while $\mathrm{BeO}$ based phosphors are highly sensitive but toxic and need encapsulation for handling $[15,16]$. The material used for encapsulation could change its energy response and it may not serve the purpose. Moreover, special techniques used for its synthesis adds to its cost. $\mathrm{Al}_{2} \mathrm{O}_{3}: \mathrm{C}$ is another good phosphor but changes its sensitivity when exposed to room light and need to be covered with black enclosures. However, it could also be optically stimulated for getting dose dependent luminescence (OSL). This technique is getting more popularity due to certain advantages. Firstly, unlike TLDs all the traps generated during irradiation are not used during readouts and the phosphor could be used again in case of any doubts in estimation of the absorbed doses or dose rates. Secondly, due to different filling rates for the multiple traps to the OSL signal resulting in complex kinetics, when irradiated with high LET charged particles, OSL decay curve shape depends upon the dose deposition profile, i.e. on both energy and LET of the particle. Therefore, when $\mathrm{Al}_{2} \mathrm{O}_{3}: \mathrm{C}$ is irradiated with a single particle type, its OSL can be used to determine the LET of the

*Corresponding author: Sahare PD, Professor, Department of Physics \& Astrophysics, University of Delhi, Delhi 110 007, India, Tel: +91-11-27667793; Fax: +91-11-27667061; E-mail: pdsahare@yahoo.co.in

Received August 03, 2012; Accepted October 05, 2012; Published October 08 , 2012

Citation: Sahare PD (2012) High-Energy Radiation Dosimetry in the Space. J Astrophys Aerospace Technol 2:e105. doi:10.4172/2329-6542.1000e105

Copyright: (c) 2012 Sahare PD. This is an open-access article distributed under the terms of the Creative Commons Attribution License, which permits unrestricted use, distribution, and reproduction in any medium, provided the original author and source are credited. 
irradiating particles as well as the absorbed dose and dose equivalent. And when irradiated in a mixed field the OSL of the phosphor can be used to determine the total absorbed dose by determining a "mean LET" value and correcting for the mean efficiency of the particles [17]. However, determination of the dose equivalent is only possible if used in combination with other detector(s), such as a plastic nuclear track detector, wherein the high-LET components (say less than $10 \mathrm{keV} / \mathrm{mm}$ ) can be separated from the low-LET components by using the data from the other detector.

Another, great advantage of OSL is that it could be used as remote detection technique. With the advent of high power LEDs and diode lasers for different optical ranges, it has become easier to stimulate the inflight/in-space (during space-walk) irradiated OSL phosphor through optical fiber online, collect the emitted light again through optical fiber, process it rapidly through online computer processors and transmit the data to the base station for further analysis. The OSL reader could be made very compact and lightweight and very low power consuming for flights and space expeditions $[18,19]$. And the whole process could be automated giving online data and alarms in case the dose exceeds the prescribed limit. It could also help for 3D mapping of the space by unmanned satellites and shuttles without risking the lives of the astronauts. However, LETs of different high-energy particles existing in the high altitudes needs to be determined using accelerator based experiments and theoretical simulations using computer codes such as Monte Carlo, On-Line Tool for the Assessment of Radiation In Space (OLTARIS), Space Ionizing Radiation Environment and Shielding Tools SIREST codes needs to be done for ready reference [5]. The guiding principle, therefore, in dealing with the radiation while working with man-made radiation sources or in the environment on the planet Earth or during the space expeditions should ensure individual dose limits applying the principle of ALARA (as low as reasonably achievable) so that individuals or groups of individuals do not get exposed exceeding the levels of acceptable risks [5]. Search for new radiation dosimeters and technologies could find the solution to this problem.

\section{References}

1. ICRP 990 recommendations of the International Commission on Radiological Protection. Pergamon press, UK.

2. Lindborg L, Bartlett DT, Beck P, McAulay IR, Schnuer K, et al. (2004) Cosmic Radiation Exposure of Aircraft Crew: Compilation of Measured and Calculated Data. Radiat Prot Dosimetry 110: 417-422.

3. Ambrozova I, Brabcova K, Spurny F, Shurshakov VA, Kartsev IS, et al. (2011) Monitoring on board spacecraft by means of passive detectors. Radiat Prot Dosimetry 144: 605-610.

4. Hallil A, Brown M, Akatov Y, Arkhangelsky V, Chernykh I, et al. (2010) MOSFET dosimetry mission inside the ISS as part of the Matroshka-R experiment. Radiat Prot Dosimetry 138: 295-309.

5. Palfalvi JK, Szabo J, Dudas B (2007) Neutron detection on the Foton-M2 satellite by a track etch detector stack. Radiat Prot Dosimetry 126: 590-594.

6. Singleterry RC, Blattnig SR, Clowdsley MS, Qualls GD, Sandridge CA, et al. (2011) OLTARIS: On-line tool for the assessment of radiation in space. Acta Astronautica 68: 1086-1097.

7. Jadrnıckova I, Tateyama R, Yasuda N, Kawashima H, Kurano M, et al. (2009) Radiat Measur 44: 901.

8. Deme S, Apathy I, Pazmandi T, Benton ER, Reitz G, et al. (2006) On-board TLD measurements on MIR and ISS. Radiat Prot Dosim 120: 438-441.

9. Fry RJM (2000) National Council on Radiation Protection and Measurements (NCRP) Report No 132

10. Caffrey JA, Hamby DM (2011) A review of instruments and methods for dosimetry in space. Adv Space Res 47: 563-574

11. Guo SL, Doke T, Zhang DH, Li L, Chen BL, et al. (2009) Study of bubble dis- tributions by high-energy protons in bubble detectors and its hints in neutron detection at higher altitude and in space. Radiat Measur 44: 885-888.

12. Green AR, Bennett LGI, Lewis BJ, Tume P, Andrews HR, et al. (2006) Characterisation of bubble detectors for aircrew and space radiation exposure. Radiat Prot Dosim 120: 485-490.

13. Ballarini F, Battistoni G, Cerutti F, Fasso A, Ferrari A, et al. (2006) GCR and SPE organ doses in deep space with different shielding: Monte Carlo simulations based on the FLUKA code coupled to anthropomorphic phantoms. Adv Space Res 37: 1791-1797.

14. Benton ER, Benton EV (2001) Space radiation dosimetry in low-Earth orbit and beyond. Nucl Instrum Methods Phys Res B 184: 255-294.

15. Yamashita T, Yasuno Y, Ikedo M (1974) Beryllium Oxide Doped With Lithium or Sodium for Thermoluminescence Dosimetry. Health Phys 27: 201.

16. Shinde SS, Dhabekar BS, Gundu Rao TK, Bhatt BC (2001) Preparation, thermoluminescent and electron spin resonance characteristics of LiF:Mg,Cu,P phosphor. J Phys D: Appl Phys 34: 2683.

17. Gaza R, Yukihara EG, McKeever SWS (2006) The use of optically stimulated luminescence from $\mathrm{Al}_{2} \mathrm{O}_{3}: \mathrm{C}$ in the dosimetry of high-energy heavy charged particle fields. Radiat Prot Dosim 120: 354-357.

18. Deneau C, Vaillé JR, Dusseau L, Mekki J (2011) Garcia P First In-Flight Data Analysis of Displacement Damage on the OSL Sensor On-Board CARMEN-2. IEEE Trans Nucl Sci 58: 939-944.

19. Gaza R, Yukihara EG, Mc Keever SWS (2008) OSU AI O :C OSL detectors, in Results from the ICCHIBAN-3 and ICCHIBAN-4 experiments to intercompare the response of space radiation dosimeters, HIMAC-128 (eds Y. Uchihori and E.R. Benton), National Institute of Radiological Sciences, Chiba, Japan. 